\title{
Using hypermedia and the Internet in the teaching of mathematics
}

\author{
Karen S. Norwood \\ North Carolina State University \\ 326 Poe Hall \\ Box 7801 \\ Raleigh, North Carolina 27695 \\ E-mail:Karen@poe.coe.ncsu.edu
}

Hypermedia documents contain links or connections to one or more other media. The advantages of hypermedia over traditional instruction are:

1. appeals to different learning styles;

2. provides a variety of means of expression for students;

3. gives students more real-world presentation experience;

4. taps into students' creativity;

5. prepares students for the 21 st century;

6. has entertainment value;

7. has storage capability.

Many educators are exploring the possibility of using the World Wide Web, not only as an information and discovery medium, but also as a tool for publishing educational material and delivering them to other educators and students in an inexpensive and efficient manner. Using the Web, educators can create maps and guide their learners through the various domains of knowledge. The Internet has two real advantages over other media. It combines the advantages of other media so that it conveys video and sound better than a book, is more interactive than a video, and, unlike CD-ROM, it can link people around the world cheaply.

Using hypermedia and the Internet enables mathematics educators to explore areas of mathematics that, without the use of the technology, would be too difficult to tackle. Secondly, it allows students to demonstrate their understanding of mathematical knowledge in creative ways through presentations and projects instead of traditional term papers. The use of these tools allows the mathematics to come to life right before students' eyes. 